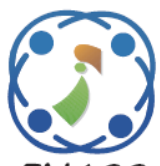

\title{
Brain Tumor Classification for MR Images using Convolution Neural Network with Global Average Pooling
}

\author{
Madhu Mallikarjuna Nayak $^{1 *} \quad$ Sumithra Devi Kengeri Anjanappa $^{2}$ \\ ${ }^{I}$ Department of Computer Science \& Engineering, GSSS Institute of Engineering \& Technology for Women, \\ Mysuru, India \\ ${ }^{2}$ Department of Information Science \& Engineering, Dayanand Sagar Academy of Technology and Management, \\ Bengaluru, India \\ * Corresponding author's Email: madhumnayak@gmail.com
}

\begin{abstract}
Magnetic Resonance Image (MRI) uses an automated system to detect the presence of tumors in brain tumor images. Many existing methods performed the classification based on machine learning approaches that include supervised and unsupervised machine learning approaches. There are various researches undergone with more efforts and many types of research related to brain tumor were examined using the small dataset. The existing models performed segmentation techniques that extracted irrelevant features showed over-fitting problems. Similarly, the segmentation process uses clustering approaches that identify the brain tumor at an early stage, which was a challenging task. Therefore, a Convolution Neural Network with Global Average Pooling (CNN-GAP) model is designed to automatically segment the tumor regions and from the segmented regions, the relevant features are extracted to overcome the problem of overfitting. The proposed CNN-GAP classifies the MRI data into Benign I and II grade or Malignant III or IV grade. The proposed CNN-GAP method showed a $2 \%$ of improvement in accuracy when compared to the existing CNN technique.
\end{abstract}

Keywords: Benign, Brain tumor, Convolution neural network global average pooling, Machine learning, Malignant.

\section{Introduction}

In the human body, the brain is an important part, which contains distinct cells with their capabilities [1]. The growth of the cell is occurred based on their functionality that loses the body capabilities and the resistance [2]. The huge cells collectively form tissues and the unusual multiplication of cells forms a tumor which would be cancerous or non-cancerous cells. These cells are classified into 4 grades of cancers that start from I to IV [3]. Grade I and II have lower grades that are referred to as Benign tumor and Grade III to IV is represented as Malignant Tumor which has high grades of tumorous cells. Detecting tumors is the foremost objective of medical researchers for decades, the progression of new treatment technology, which requires more time and good finance [4]. The field of Medical Science is a core technology that analyses the tumor and is also used to find the safer method for curing all causes. Imaging techniques like Magnetic Resonance Imaging (MRI), Computed Tomography (CT), and Positron Emission Tomography (PET) are widely used to detect brain tumors due to their imaging principle that depends on the resolution of the spatial intensities [5]. Moreover, it provides detailed information about brain anatomy, vascular supply, and cellular structure which is an important tool for effective tumor monitoring, diagnosis, and treatment [6]. These MRI brain images are used to segment the tumor portions which is an interesting and emerging research area in the field of medical imaging. The main objective of the research is to store medical images in the distributed environment to process in parallel computing to unleash the hidden and relevant information. There is a requirement to deal with large-scale medical images to obtain better and accurate classification results [7]. Big Data Analytics 
is performed for massive analyses to perform discriminative tasks with the data volumes to perform prediction and classification [8-10]. These existing researches underwent brain tumour segmentation, which is described in the next section.

The structure of the paper is as follows: in Section 2 the literature review for the existing methods is discussed, Section 3 describes the proposed CNNGAP algorithm. Section 4 describes the results and discussion which are evaluated quantitatively and comparatively. Finally, Section 5 discussed the conclusion and future work.

\section{Literature review}

The review on the existing methods for brain tumour detection is as follows:

Jose Dolz [11] developed Ensemble semi-densed fully connected CNNs for Brain tumor classification. The developed model showed an efficient propagation during data training that limited the number of parameters due to less generation of magnitude using 3D U-Net. However, the developed model faced difficulty for the ground truth labels to test the participated teams for an instance.

Dong Nie [12] developed a 3D Fully CNN that integrated the dense features and coarse features that were considered to improve the performances for segmentation. The hierarchical features generated were aggregated that speed up the network convergence and achieved the highest accuracy. However, the developed CNN model localized the local image patch but the text information was missed that failed for guiding the tissue segmentation and spatial consistency showed difficulty in obtaining results during interpretation.

Gerard Sanroma [13] developed a Multi -atlas label fusion Stacking Cascading Segmentation. The results obtained from the developed model showed that the learning capabilities from the systematic combination showed performance improvement as the model utilized individual methods for enhancing the learning capabilities. The developed model performed successfully combined the stacks based on the combination strategy which showed improvement in the performance but when cascaded it failed to show improvement as more levels of features were added.

Raymond Pomponio [14] developed Multi-Atlasbased image processing techniques based on a webbased visualization interface which generated and presented the resulting age trends for the brain structure. However, the developed model required to focus on other framework applications to other brain datasets, variables of interests that volunteered the patients based on the derivation of disease-specific trends.

Yunjie Chen [15] developed a Gaussian mixture model (GMM) that used spatial factor that was not considered the local neighbouring information, but also considered the pixels to extract the spatial information. The Mutual Information (MI) identified and eliminated the outliers during brain tumour classification. The classical GMM considered only the intensity distribution that created noises in the data without spatial information.

Abolfazl Kouhi [16] developed a Fuzzy C-Means (FCM) clustering algorithm that solved the drawbacks obtained for spatial constraints which are ineffective in preserving the details of the image. Continuously, the approach resulted in noises and maintained the Intensity Non-Uniformity (INU). However, the model could not overcome the problem of computational complexity when compared with other clustering approaches as the membership matrix was updated twice in each iteration through spatial constraints.

Narayana [17] developed a DL based on Segmentation approaches for brain tumor detection. The segmentation process obtained a better accuracy to train the size that has to determine the function. The data were fitted with a learning curve that estimated the data training size to determined better accuracy. However, the power-law dependence showed a better prediction of up to 50 images, which are required to given target was absent.

\section{Proposed method}

The block diagram of the proposed method is shown in Figure 1. Initially, the database was taken from the Standard MRI brain tumor image database to conduct the proposed work. The extracted image data must undergo data pre-processing and perform Segmentation for obtaining the brain tumor segments. The classification results showed that two classes are obtained once after using the classifier into Benign and Malignant. An elaborate explanation for the modified CNN in the present research work is explained in the upcoming contexts.

\subsection{MRI brain tumor database}

The brain tumor dataset consists of $3064 \mathrm{~T} 1$ weighted contrast-enhanced images of 233 patients who suffer from any of the three stages (1st stage, 2 nd stage, and 3rd stage) of the brain tumor. The dataset images are taken from the hospitals of China like Nanfang Hospital and General Hospital has the record which starts from 2005 [18]. The dataset 


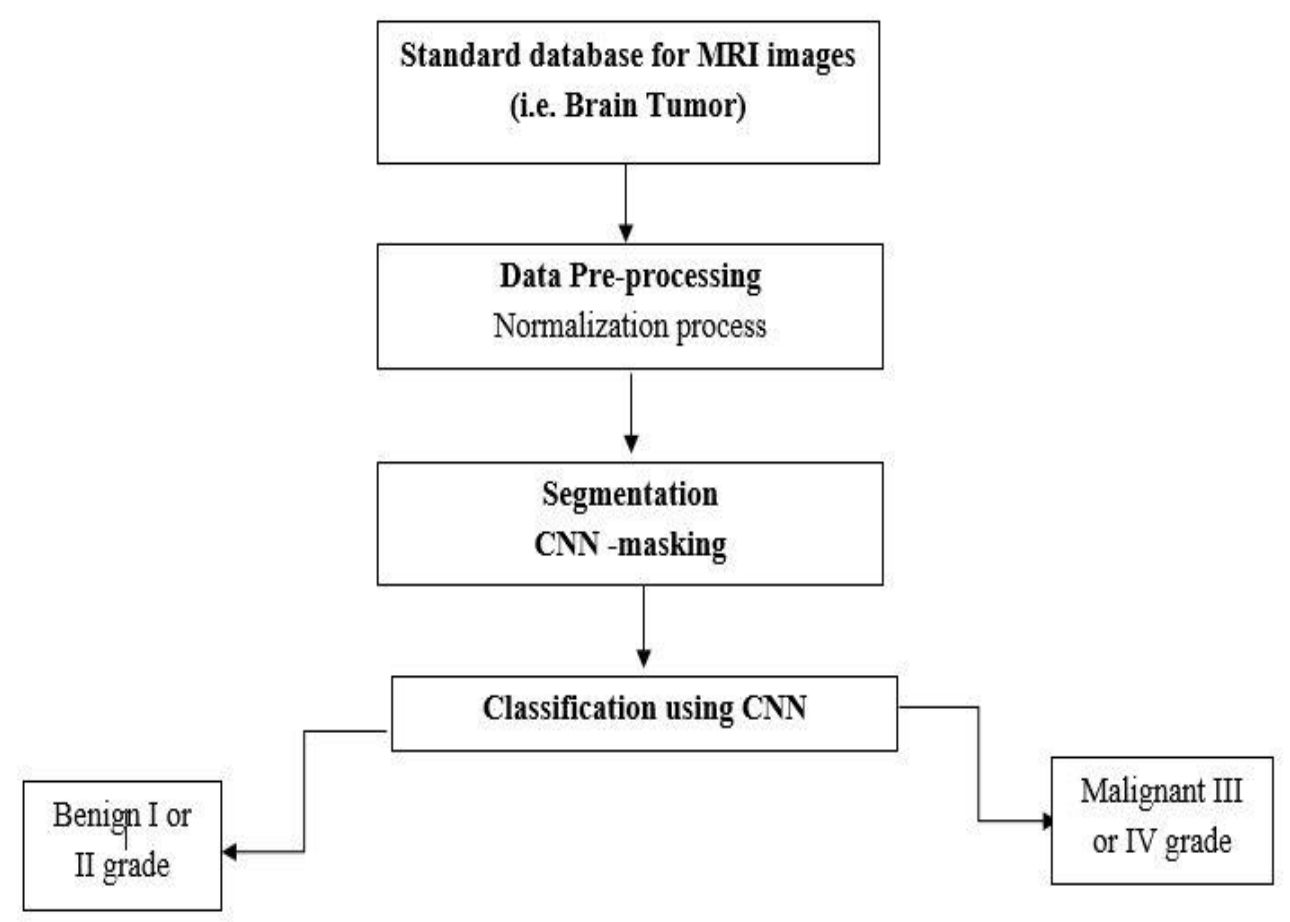

Figure. 1 Block diagram of the proposed method
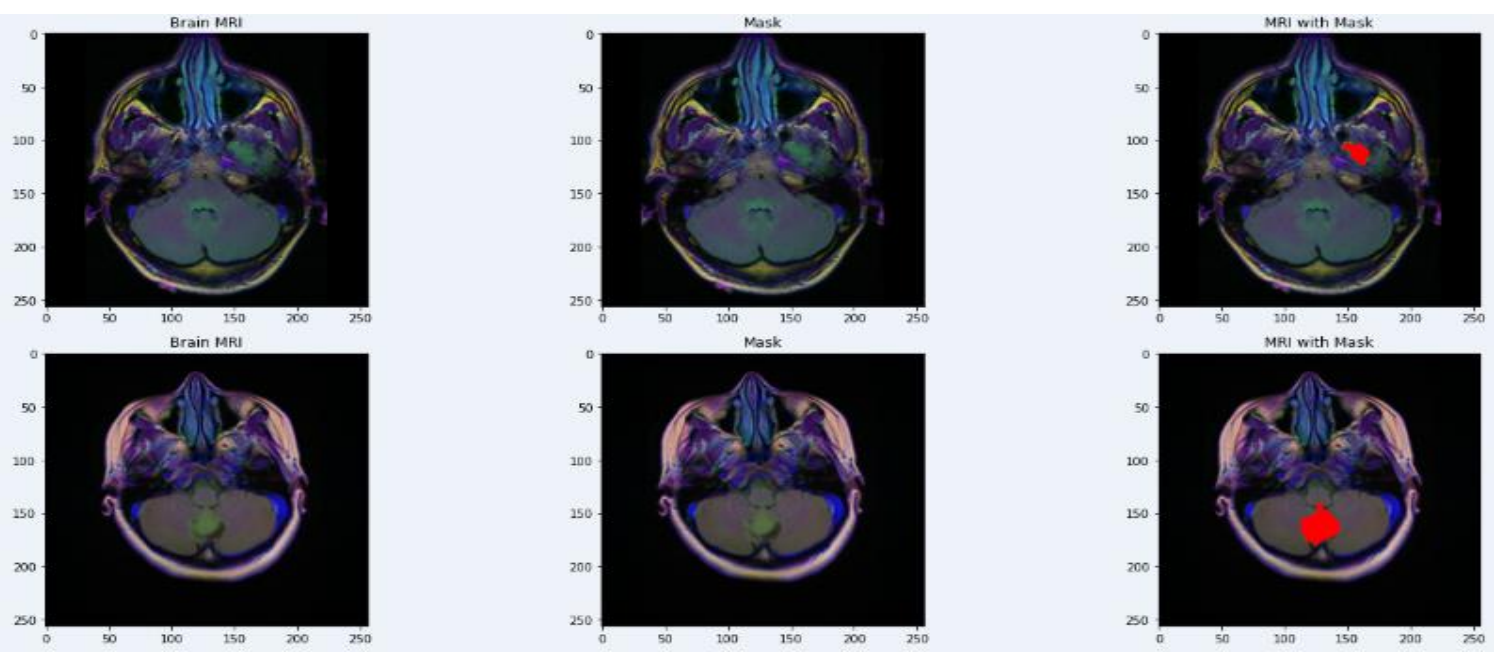

Figure. 2 MRI brain tumor database

images were applied for the normalization process and thus helped the model for performing the best. This present section utilizes a brain MRI source of image dataset which is used to perform the segmentation of MRI. The test images are acquired with TR which utilizes 5850 and Echo time as 130. The FLAIR weighted MRI was acquired to the spectra MR machine with 135 images in total with 9 slices of image per patient and each of the images having FOV of $200 \mathrm{~m}$. The real dataset including the brain MRI has a $512 \times 512$ pixel size which is converted to grayscale before processing. In the proposed method, the large dataset is used to overcome the problem by using a small dataset for misclassification in existing CNN methods. Figure 2 shows the sample MRI brain tumor images present in the database.

\subsection{Pre-processing using Min-Max normalization}

The normalization is performed using the Minmax normalization process, which consists of rescaling the intensity value of interest by deducting the minimum value. The different augmentation techniques maximize data runtime, which generalizes the model to achieves high results. Thus, the pre- 
processing step enhances the images that overcome the obstacles or noises in the images and undergone for segmentation process. The examination of a large number of patient's data is considered to the present research which accurately determines the brain tumor. The missing data in a large dataset occurs due to human error, database failure, or malfunctioning of the system. An incomplete and uncertain medical data is present in an image data that should be removed for improving the quality of an image. Next, the integration of data is concluded using data preprocessing. The Min-Max normalization process plays an important role in the integration and as well as data normalization. Every feature value has a minimum value which gets transformed into 0 and the maximum value is transformed into 1 . Both values are converted into decimals that are range from 0 and 1. Eq. (1) expresses the normalization process.

$$
X_{\text {norm }}=\frac{X_{i}-X_{\min }}{X_{\max }-X_{\min }}
$$

Where, $X_{i}$ is the $i^{\text {th }}$ data point, $X_{\text {min }}$ is the minimum value of the data point, $X_{\max }$ is the maximum value of the data point or the batch instances $\mathrm{D}=\left\{\mathrm{X}_{0}, \ldots . \mathrm{X}_{\mathrm{n}-1}\right\}$. These variables calculate the normalized value which is known as $\mathrm{X}_{\text {norm }}$. The normalized data obtained is now in structure format that fills with the missing data. The segmentation technique becomes an important task when the huge complex images are required to generate the region of interest based on the brain's anatomical structure. The process is used to overcome noisy images, low contrast, hidden and inaccurate boundary of an organ. Initially, the neighborhood pixels are examined at the seed point to determine whether the pixel neighbor is required to be added to mask the brain region. Especially, the masking based on CNN preserves brain image pixels applied to all types of brain tissue to accurate determination of cancer. The brain mask segmentation is performed by computing 2 images $T_{1 w}$ and $T_{2 w}$ for brain mask segmentation given as follows,

Step 1: The resampled $T_{2 w}$ image is matched by the resolution of the $T_{1 w}$ image.

Step2: The voids are eliminated subsequently during foreground segmentation of the $T_{2 w}$ image which is shown in Eq. (2)

Step 3: The maximum intensity is achieved by matching the rescaling of the $T_{1 w}$ image with the intensity of the closed $T_{2 w}$ image is calculated using Eq. (3)

Step 4: The segmentation of the foreground image is obtained from the background image and the
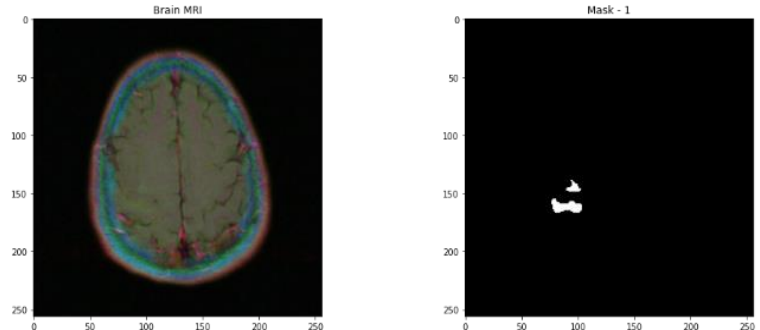

Figure. 3 The left image is the brain MRI image and right side of the image is the masking of tumor region
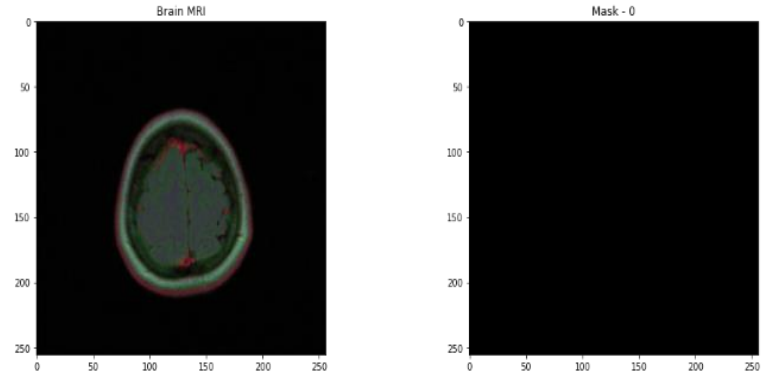

Figure. 4 The left image is the brain MRI image and the right side of the image has no mask

output image of region growing is presented in Fig. 3 and 4.

The weighted within the class probabilities are calculated using the below Eq. (2)

$$
\begin{gathered}
q_{1}(t)=\sum_{i=1}^{t} P(i), q_{2}(t) \\
=\sum_{i=t+1}^{I} P(i) q_{n}(t) \\
=\sum_{i=I+t+1}^{n} P(i)
\end{gathered}
$$

The threshold value ranges from 1 to $t$

$q_{1 . ., n}$ are weighted class within pixel probabilities $P$ of the foreground and background. The class means for $T_{1 w}$ image with the intensity of the closed $T_{2 w}$ is given as (3)

$$
\begin{gathered}
\mu_{1}(t)=\sum_{i=1}^{t} \frac{i P(i)}{q_{1}(t)}, \mu_{2}(t)= \\
\sum_{i=t=1}^{I} \frac{i P(i)}{q_{2}(t)}, \ldots ., \mu_{n}(t)=\sum_{i=n}^{t} \frac{i P(i)}{q_{n}(t)}
\end{gathered}
$$

$\mu_{1}$ and $\mu_{2}$ are the average gray level values, $I$ is the maximum intensity. The segmented mask region with structural element is given as input image which produces the same size of output image without losing their qualities. In this research work, the objective is to deal with large-scale medical images which obtain better and accurate classification results.

\subsection{Classification using CNN with global average pooling}

The CNN is a type of neural network that considers the structures like spatial pooling layers, 


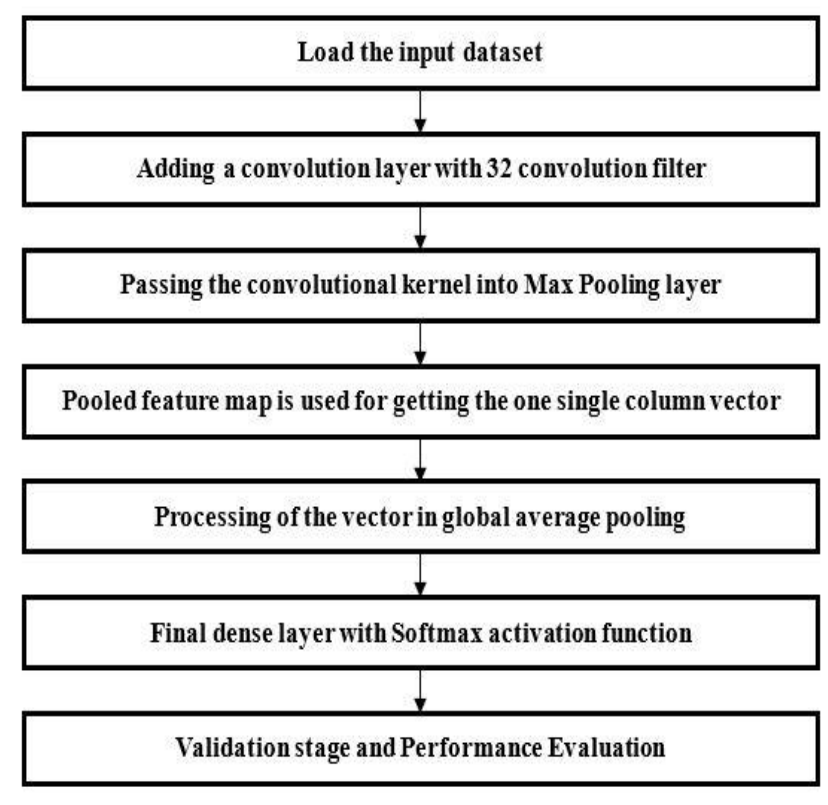

Figure. 5 Flow chart for CNN model

convolution layers, fully connected layers, and local response normalization layers. A novel stacked CNN model with fully connected Convolution layers has decreases the filter sizes that are used to improve the performance of proposed CNN-GAP models. CNN is a type of discriminate deep architecture that consists of a Convolution layer and a pooling layer that is stacked with one another on top forms a deep model.

The GAP layer allocates the weight and the subsamples from the pooling layer generate an output in the Convolution layer that reduces the data rate for the layer present below. Additionally, the ensemble stack model improves the diagnostic quality and statistical measures of the performance, when it comes to decision making in the biomedical field. The classification results showed that the brain MRI is of Malignant or Benign type of brain tumor. The flow chart for the CNN model is as shown in Figure 5. The ConvNets are created often which continues to be as a hand-in-hand approach that reaches up to the pooling layers. The additional layers like average pooling, max pooling, and global pooling layers are constructed in the network layer. The pooling operations are performed to the conceptual level and the inner working layer ConvNet analyzes the pooling layers, which are similar to that of the spatial hierarchy models. The pooling such as average pooling, max pooling, global max pooling, and global average pooling is continued with the identification process. The pooling layers are represented within Keras models which are having the most wider DL frameworks. The other pooling layer is known as the Global Max Pooling layer is set with pool size which is equal to input size and the maximum of the entire input is computed with the output value. The GAP pooling operation is designed which is replaced to fully connected layers using the classical CNNs. In each layer, the feature map corresponds to that of the classification performs the task using Multi-Layer Perceptron (MLP) Convolution layer. Alternatively, the connected layers are added to the top of feature maps as the average obtained from each feature map will be a vector directly fed for the Softmax layer. In the architecture, the GAP layers are designed, consist of a final max-pooling layer that included an activation map in the dataset to each image category. The GAP layer obtained vectors that are fed to the max-pooling layer has the possibility for each of the objects during the process of classification. The Softmax activation function obtained better probability results for each class and the proposed GAP-CNN showed that in an image, the tumor region was present. It also It is stated that each object in an image was not a part of the additional work. The localization process is performed to heat the map where the color coding scheme was identified in the region that was relatively important to perform GAP$\mathrm{CNN}$ for the identifying of an object and the CNNGAP model is shown in the figure 6. Activation maps are presented to act as a detector for determining the pattern to perform the localization which is appeared on image space. An activation map for each class corresponds to an image that detected the objects and patterns. The transformation of the patterns notices GAP layers which generates an activation maps corresponded to the weights which are encoded using activation map functions. The sum of the contributions obtained from the detected patterns is generated activation maps that detected the object classes which have more weight.

The GAP constitutes of the first sub layer is a 1D local max pooling with a window size of $q$ with zero paddings, and the output length is $\frac{1}{q}$ of the

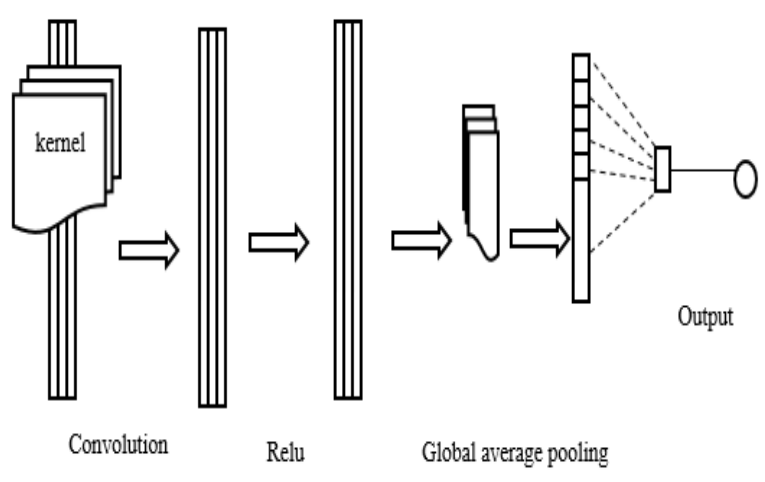

Figure. 6 Proposed CNN-GAP 
original length. Each layer calculates the weights using the Eqs. (4) to (7) is shown as follows:

\section{The input of Global pooling:}

$$
\text { Input }=A_{i}
$$

Where, $i=1, \ldots, L$

The first sub - Layer $\left(B_{i}\right)$ :

$$
\begin{gathered}
B_{i}=\min _{k=0,1, \ldots, q-1}\left\{A_{q i-k}\right\}, \\
\text { Where } i=1, \ldots, \frac{L}{q}
\end{gathered}
$$

\section{Second sub layer:}

$$
\text { Output }=\sum_{i}^{\frac{L}{q}} w_{i} B_{i}
$$

Where,

$$
w_{i}=\frac{1}{z} \exp \left\{m\left(B_{i}-\bar{B}\right\}\right.
$$

Where $\exp \left\{m\left(B_{i}-\bar{B}\right\}, m>0, \bar{B}\right.$ refers to average scores obtained from the 4 layers. The hyper parameters $m$ and $q$ are determined by the validation. $L$ is the penalty added for the weights that help to train the model, which is assigned to the insignificant input features that are helpful by avoiding overfitting with zero padding could be done at the end of $A_{i}$. The table 1 shows the parameter settings for the proposed CNN-GAP that describes about the layer and their dimensions.

\section{Pseudo code for CNN-GAP}

Step 1: These four layers like convolution, ReLU, GAP, and output are working in parallel to extract the different levels of features,

\#The individual convolution layer is accompanied by batch normalization and ReLU layers.

Step 2: The output of four branches is merged in the concatenation layer, and then it is pushed to the second block.

Step 3: In the end, the GAP layer reduces the whole height and weight to one vector which is a high dimensional reduction overfitting.

\#The model has less chance to gain

Step 4: Fully connected layer is employed to the output layer.
Table 1. Parameter settings for CNN-GAP

\begin{tabular}{|c|c|}
\hline Layer & Dimension \\
\hline Input Layer & $224 \times 224 \times 3$ \\
\hline Convolution2D & $224 \times 224 \times 64$ \\
\hline Maxpooling Layer & $112 \times 112 \times 64$ \\
\hline Convolution2D & $112 \times 112 \times 128$ \\
\hline Maxpooling Layer & $112 \times 112 \times 128$ \\
\hline Convolution2D & $56 \times 56 \times 256$ \\
\hline Maxpooling Layer & $56 \times 56 \times 256$ \\
\hline Convolution2D & $28 \times 28 \times 512$ \\
\hline Maxpooling Layer & $28 \times 28 \times 512$ \\
\hline Convolution2D & $14 \times 14 \times 512$ \\
\hline Maxpooling Layer & $14 \times 14 \times 512$ \\
\hline Convolution2D & $14 \times 14 \times 512$ \\
\hline Maxpooling Layer & $7 \times 7 \times 512$ \\
\hline Global Average Pooling layer & 512 \\
\hline Dense layer & 1024 \\
\hline Dense layer & 512 \\
\hline Dense layer & 2 \\
\hline
\end{tabular}

An advantage of the global average pooling consisted of fully connected layers which are similar to the convolution structure to enforces the correspondence among the categories and the feature maps. The feature maps are interpreted easily to the categories, maps the confidence. By optimizing the parameter with GAP avoids the layers from overfitting problems as GAP sums all the spatial information. The proposed CNN-GAP classifies the MRI image into Benign I and II grade or Malignant III or IV grade resulting images are shown in figure 7. In Figure 7, Fig. 7(a) is the Brain original MRI, Fig. 7(b) is the Original Mask obtained for the brain image, Fig. 7(c) is the Predicted Mask region obtained for the MRI, Fig. 7(d) is the MRI with original Mask and Fig. 7 (e) is the MRI with predicted Mask.

\section{Results}

The proposed model evaluated the results of classification using accuracy, F1-score, recall, precision indicated a clear generalization of the model that imbalanced the MRI images dataset. The metrics depend on the class imbalance and the overall model performance is required concerning the class count. The simulations are conducted using an Intel Core i7 processor with $2 \mathrm{GHz} \mathrm{CPU}$ utilization time and $48 \mathrm{~GB}$ of RAM. The training data is fed to the classifier and evaluated with respect to the testing data. The achieved performances such as Accuracy, Precision and Recall are described as follows and are calculated using the Confusion matrix which is shown in Figure 8. 

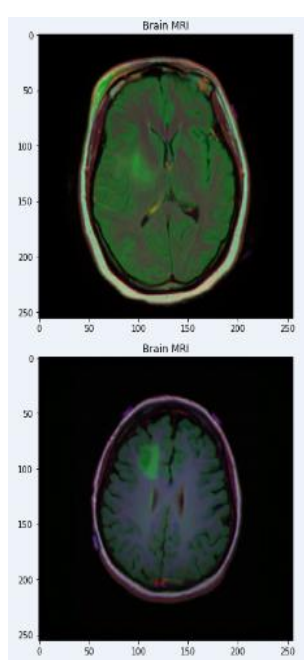

(a)
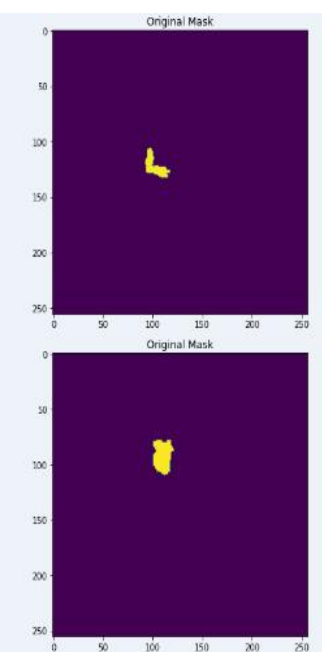

(b)
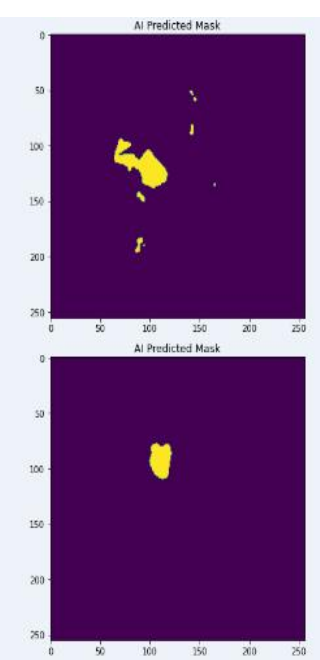

(c)
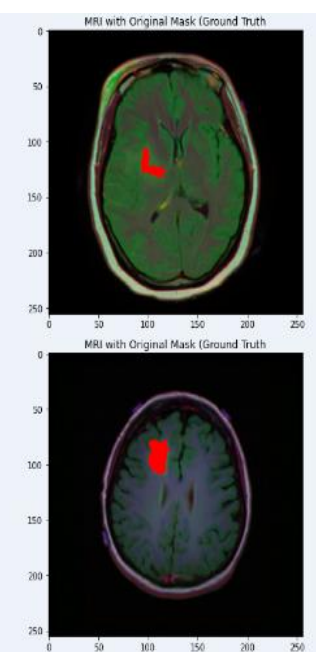

(d)
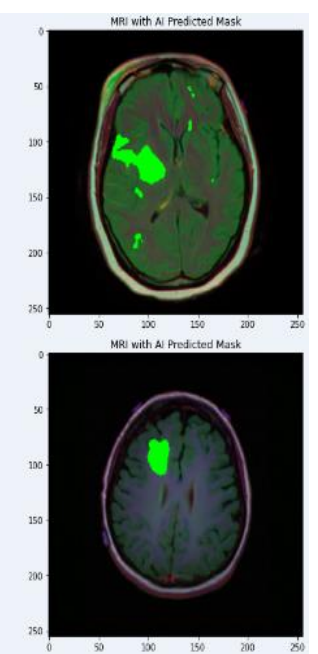

(e)

Figure. 7 (a) Brain original MRI, (b) Original mask, (c) Predicted mask, (d) MRI with original mask, and (e) MRI with predicted mask

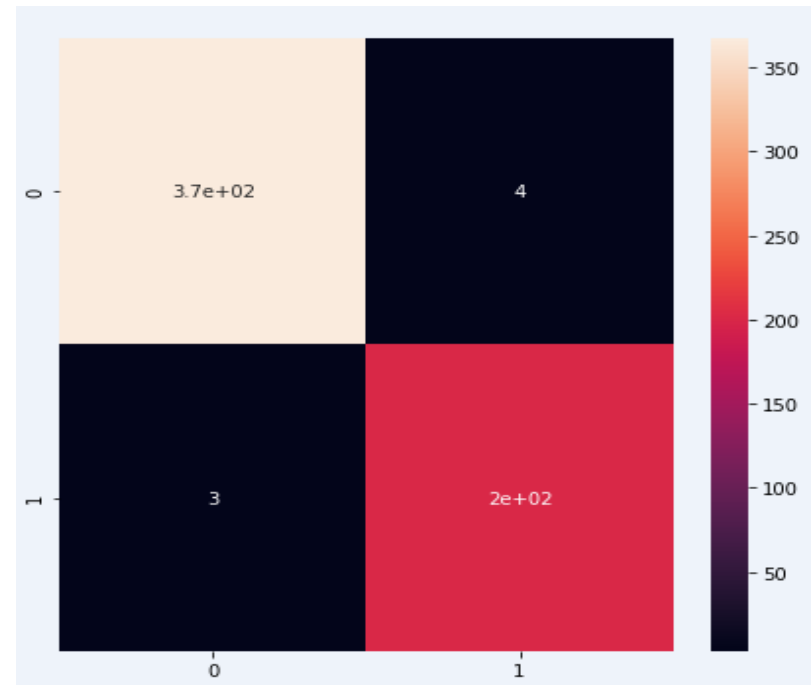

Figure. 8 Confusion matrix

\subsection{Performance measures}

\subsubsection{Accuracy:}

Accuracy is defined as the ratio of correctly predicted to the total number of observations. The accuracy is calculated by using Eq. (8)

$$
\text { Accuracy }=\frac{(T P+T N)}{(T P+T N+F P+F N)}
$$

\subsubsection{Precision}

Precision is defined as the total number of data samples predicted observations as false to the actual number of false observations. The mathematical Eq. (9) describes the precision.

$$
\text { Precision }=\frac{T P}{T P+F P}
$$

\subsubsection{Recall}

When the actual number of traditional false observations are considered as the ratio of exactly predicted as false observations are defined as recall. The proportion of actual positives is predicted by using recall, which is shown in Eq. (10):

$$
\text { Recall }=\frac{T P}{T P+F N}
$$

\subsubsection{F1-measure}

The harmonic mean of recall and precision is defined as F1-Measure, which is shown in Eq. (11).

$$
F 1-\text { measure }=\frac{2 \times \text { Precision } \times \text { Recall }}{\text { Precision }+ \text { Recall }}
$$

\subsection{Quantitative analysis}

Table 2 shows the values obtained for the proposed CNN-GAP technique. The results of the proposed CNN-GAP are evaluated in terms of Accuracy and Precision. The support values for class type 0 is 371 and the support value for class 1 is 205 . The precision value of 0.99 is obtained for the class type 0 and 0.98 for the class type 1 which is shown in table 1 . The graphical representation of the proposed CNN-GAP is shown in Figure 9. The results for the proposed CNN-GAP are evaluated in terms of Recall and F-score. The recall value of 0.99 is obtained for 
Table 2. Quantitative analysis for the proposed CNN-

\begin{tabular}{|c|c|c|c|}
\hline $\begin{array}{c}\text { Class } \\
\text { type }\end{array}$ & Precision & Recall & F-score \\
\hline 0 & 0.99 & 0.99 & 0.99 \\
\hline 1 & 0.98 & 0.98 & 0.98 \\
\hline
\end{tabular}

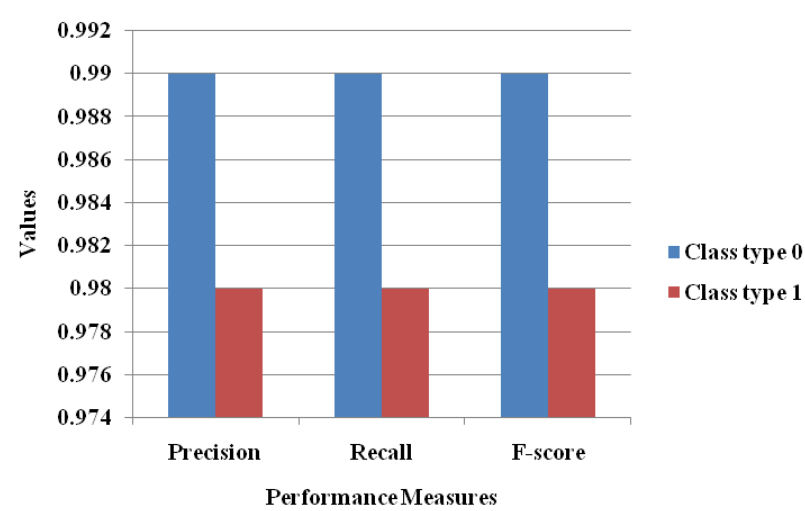

Figure. 9 Graphical representations for the proposed method in terms of Precision, Recall and F-score

Table 3. Results obtained for CNN-GAP

\begin{tabular}{|c|c|c|c|}
\hline Class type & Precision & Recall & F-score \\
\hline 0 & 0.99 & 0.99 & 0.99 \\
\hline 1 & 0.99 & 0.99 & 0.99 \\
\hline Avg. & 0.99 & 0.99 & 0.99 \\
\hline
\end{tabular}

the class type 0 and 0.99 value which is obtained for the class type 1 . The F-score value of 0.99 is obtained for the class type 0 and the 0.98 value is obtained for the class type 1 .

Table 3 shows the values obtained for the proposed CNN-GAP technique. The results for the proposed CNN-GAP are evaluated in terms of Precision, Recall and F-score. The recall value of $0.99 \%$ is obtained for class-type 0 and the 0.99 value is obtained for class type 1 to Macro Average. An Fscore value of 0.99 is obtained for the class type 0 and a 0.99 value is obtained for the class type 1 . The weighted average value of 0.99 is obtained for precision, recall, and F-score is represented as shown in Fig. 2.

\subsubsection{Comparative analysis}

The proposed CNN-GAP and the comparative analysis with the existing methods are shown in Table 4.

The accuracy for the existing [11] 3D-CNN with ensemble model obtained $96 \%$ and Error Value of $4 \%$. Similarly, [12] developed 3-D fully CNN achieved an accuracy of $92.69 \%$ and 7.31 with respect to $3064 \mathrm{~T} 1$-weighted contrast-enhanced brain tumor images. Yunjie Chen [15] developed GMM model that obtained accuracy ranging from $78.22 \%$
Table 4. Accuracy and error values obtained for the proposed and existing methods

\begin{tabular}{|c|c|c|c|}
\hline Authors & Methodology & $\begin{array}{c}\text { Accuracy } \\
(\mathbf{\%})\end{array}$ & $\begin{array}{c}\text { Error } \\
\text { Values }\end{array}$ \\
\hline Dolz [11] & $\begin{array}{c}\text { 3D CNN, } \\
\text { ensemble } \\
\text { learning. }\end{array}$ & 96 & 4 \\
\hline Nie [12] & $\begin{array}{c}\text { 3-D Fully } \\
\text { CNN }\end{array}$ & 92.69 & 7.31 \\
\hline $\begin{array}{c}\text { Yunjie Chen } \\
\text { [15] }\end{array}$ & GMM & $\begin{array}{c}78.22 \text { to } \\
91.09\end{array}$ & $\begin{array}{c}21.78 \text { to } \\
8.91\end{array}$ \\
\hline $\begin{array}{c}\text { Abolfazl } \\
\text { Kouhi [16] }\end{array}$ & FCMS-MLI & 94.38 & 5.62 \\
\hline $\begin{array}{c}\text { Ponnada A. } \\
\text { Narayana } \\
{[17]}\end{array}$ & Fully CNN & 86 to 91 & 14 to 9 \\
\hline Proposed & CNN-GAP & 98.78 & 1.22 \\
\hline
\end{tabular}

to $91.09 \%$ and Error Value ranging from 21.78 to 8.91. Abolfazl Kouhi [16] develoepd FCMS-MLI model that achieved accuracy of 94.38 and Error Value of 5.62. Ponnada A. Narayana [17] developed Fully connected CNN model that obtained accuracy ranging from 86 to 91 and Error Values ranging from 14 to 9 . The existing models faced difficulty for the ground truth labels when cascaded for improvement in the performance failed to show improvement as more levels of features were added was difficult during segmentation. Whereas, the segmented mask region with structural element was given as input image which produced the same size of output image without losing their qualities. The proposed CNNGAP model was designed that automatically segmented the tumor regions and from the segmented regions, the relevant features extracted overcame the problem of overfitting. The proposed CNN-GAP obtained an accuracy of $98.78 \%$ and error value of 1.22 that showed better improvement in the performance when compared with the existing methods.

\section{Conclusion}

Currently, the research work performs learning approaches through sequential stages by initializing with pre-processing, feature extraction, feature selection process and classification process that obtains final output. The existing models have examined various machine-learning techniques to classifying brain tumors. They face challenges during the classification of a small dataset and training irrelevant features, which shows the misclassification. Therefore, the proposed model used a large dataset for the classification that was rarely available. The small datasets used for the proposed CNN-GAP research are automatically performed by 
segmentation where the relevant features were extracted to overcome the over-fitting problem. The proposed method shows a $2 \%$ improvement in accuracy when compared with the existing CNN technique. However, in the future, complexity is created in the CNN network due to more number of layer parameters that should be overcome by integrated models. These integrated models should be improved for all the modalities of the MRI images in segmentation of the tumors.

\section{Conflicts of Interest}

The authors declare no conflict of interest.

\section{Author Contributions}

The paper background work, conceptualization, methodology, dataset collection, implementation, result analysis and comparison, preparing and editing draft, visualization have been done by first author. The supervision, review of work and project administration, have been done by second author.

\section{References}

[1] M. Sajjad, S. Khan, K. Muhammad, W. Wu, A. Ullah, and S. W. Baik, "Multi-grade brain tumor classification using deep CNN with extensive data augmentation", Journal of Computational Science, Vol. 30, pp. 174-182, 2019.

[2] Z. N. K. Swati, Q. Zhao, M. Kabir, F. Ali, Z. Ali, S. Ahmed, and J. Lu, "Brain tumor classification for MR images using transfer learning and finetuning", Computerized Medical Imaging and Graphics, Vol. 75, pp. 34-46, 2019.

[3] N. Ghassemi, A. Shoeibi, and M. Rouhani, "Deep neural network with generative adversarial networks pre-training for brain tumor classification based on MR images", Biomedical Signal Processing and Control, Vol. 57, p. 101678, 2020.

[4] H. H. Sultan, N. M. Salem, and W. A. Atabany, "Multi-Classification of BrainTumor Images Using Deep Neural Network", IEEE Access, Vol. 7, pp. 69215-69225, 2019.

[5] K. Kaplan, Y. Kaya, M. Kuncan, and H. M. Ertunç, "Brain tumor classification using modified local binary patterns (LBP) feature extraction methods", Medical Hypotheses, p. 109696, 2020.

[6] I. E. Kaya, A. Ç. Pehlivanlı, E. G. Sekizkardeş, and T. Ibrikci, "PCA basedclustering for brain tumor segmentation of T1w MRI images", Computer Methods and Programs in Biomedicine, Vol. 140, pp. 19-28, 2017.
[7] S. A. A. Ismael, A. Mohammed, and H. Hefny, "An enhanced deep learningapproach for brain cancer MRI images classification using residual networks", Artificial Intelligence in Medicine, Vol. 102, pp. 101779, 2020.

[8] G. Tamilmani, and S. Sivakumari, "Early detection of brain cancer using association allotment hierarchical clustering", International Journal of Imaging Systems and Technology, Vol. 29, pp. 617-632, 2019.

[9] S. Maharjan, A. Alsadoon, P. W. C. Prasad, T. A. Dalain, and O. H. Alsadoon, "Anovel enhanced softmax loss function for brain tumour detection using deep learning", Journal of neuroscience methods, Vol. 330, p. 108520, 2020.

[10] S. Deepak and P. M. Ameer, "Brain tumor classification using deep $\mathrm{CNN}$ featuresvia transfer learning", Computers in Biology and Medicine, Vol. 111, p. 103345, 2019.

[11] J. Dolz, C. Desrosiers, L. Wang, J. Yuan, D. Shen, and I. B. Ayed, "Deep CNN ensembles and suggestive annotations for infant brain MRI segmentation", Computerized Medical Imaging and Graphics, Vol. 79, p. 101660, 2020.

[12] D. Nie, L. Wang, E. Adeli, C. Lao, W. Lin, and D. Shen, "3-D fully convolutional networks for multimodal isointense infant brain image segmentation", IEEE transactions on cybernetics, Vol. 49, pp. 1123-1136, 2018.

[13] G. Sanroma, O. M. Benkarim, G. Piella, K. Lekadir, N. Hahner, E. Eixarch, and M. A. G. Ballester, "Learning to combine complementary segmentation methods for fetal and 6-month infant brain MRI segmentation", Computerized Medical Imaging and Graphics, Vol. 69, pp. 5259, 2018.

[14] R. Pomponio, G. Erus, M. Habes, J. Doshi, D. Srinivasan, E. Mamourian, V. Bashyam, I. M. Nasrallah, T. D. Satterthwaite, Y. Fan, and L. J. Launer, "Harmonization of large MRI datasets for the analysis of brain imaging patterns throughout the lifespan", NeuroImage, Vol. 208, p. 116450, 2020.

[15] Y. Chen, M. Cai, X. Zhou, C. Ning, C. Cao, and J. Yang, "A Robust Spatial InformationTheoretic GMM Algorithm for Bias Field Estimation and Brain MRI Segmentation", IEEE Access, Vol. 8, pp. 89617-89629, 2020.

[16] A. Kouhi, H. Seyedarabi, and A. Aghagolzadeh, "Robust FCM clustering algorithm with combined spatial constraint and membership matrix local information for brain MRI segmentation", Expert Systems with Applications, Vol. 146, pp. 113159, 2020. 
[17] P. A. Narayana, I. Coronado, S. J. Sujit, J. S. Wolinsky, F. D. Lublin, and R. E. Gabr, "Deeplearning-based neural tissue segmentation of MRI in multiple sclerosis: Effect of training set size", Journal of Magnetic Resonance Imaging, Vol. 51, pp. 1487-1496, 2020.

[18] S. Bhuvaji, A. Kadam, P. Bhumkar, S. Dedge, and S. Kanchan, "Brain Tumor Classification (MRI)", Dataset, Available online: https://www.kaggle.com/sartajbhuvaji/braintumor-classification-mri (accessed on 1 August 2020). 